\title{
Enzymatic Conversion of Heme to Bilirubin in Normal and Starved Fetuses and Newborn Rats
}

\author{
M. Michael Thaler ${ }^{[25]}$, David L. Gemes, and Arne F. Bakken \\ Departments of Pediatrics and Medicine, University of California, San Francisco, California, USA
}

\begin{abstract}
Extract
Heme oxygenase ( $\mathrm{HO})$, the enzyme system responsible for the conversion of heme to bilirubin, has an average activity of $0.143 \pm 0.036 \mathrm{~m} \mu$ moles bilirubin/ $10 \mathrm{mg}$ protein/ min in fetal rat liver between 15 and 21 days of gestation, compared with $0.070 \pm$ 0.020 in mature liver. Heme oxygenase activity increases rapidly during the first postnatal day $(0.237 \pm 0.078)$ and reaches 4 times adult rates at 1 week of age. Thereafter, activity declines to basal adult rates at weaning. Splenic $\mathrm{HO}$ activity is relatively low during the fetal and immediate postnatal period compared with its activity in mature animals (0.206 versus 0.790$)$, and rises to nearly 11 times the hepatic activity in mature animals.

Fasting of pregnant rats for 3 days before delivery caused a $20 \%$ average increase in hepatic HO of fetuses and newborns. In newborn rats fasted for $3 \mathrm{hr}$ and $6 \mathrm{hr}, \mathrm{HO}$ activity increased two- and threefold, respectively, compared with fed controls. Refeeding after $3 \mathrm{hr}$ resulted in a decline to basal values at $6 \mathrm{hr}$ of age. Splenic activity was not affected by fasting or refeeding.

These results show that the capacity of the liver for bilirubin production is greater in fetuses compared with adults, and increases further at birth. Starvation in mother or offspring stimulates the hepatic enzymic process for conversion of heme to bilirubin.
\end{abstract}

\section{Speculation}

These studies should provide a basis for investigating nutritional and hormonal factors which influence the conversion of heme to bilirubin in fetuses and newborns.

\section{Introduction}

Several lines of evidence suggest that relatively large amounts of heme are catabolized to bilirubin in near term fetuses and newborn infants $[3,10,20]$. In addition, blood group incompatibility is a frequent cause of severe hemolysis before and after birth. The enzymatic conversion of large amounts of heme to bilirubin in newborn infants who are deficient in eliminating the pigment results in unconjugated hyperbilirubine- mia which may cause kernicterus or other toxic complications. For these reasons, the capacity of the fetus and newborn for heme catabolism is of major clinical interest.

Tenhunen, Marver, and Schmid [15] recently described microsomal heme oxygenase, the enzyme system responsible for the conversion of heme to bilirubin. In mature rats, heme oxygenase activity is greatest in liver and spleen [17]. Hepatic heme oxygenase in rats has been shown to increase following injections of 
hemoglobin or heme (methemalbumin) [17], in experimental hemolytic anemia [17], and during periods of starvation $[1,2]$. This report concerns the development of heme oxygenase in fetal and newborn rat liver and spleen, the effects of starvation and refeeding on heme oxygenase activity in newborns, and the influence of maternal starvation on heme oxygenase in offspring before and after birth.

\section{Materials and Methods}

All experiments were performed on Sprague-Dawley rats. When fetuses were investigated, pregnancies were timed to within $12 \mathrm{hr}$ of conception. Pregnant rats had free access to Berkeley diet laboratory chow and water. The effects of maternal starvation on the fetus were examined by fasting pregnant animals for $72 \mathrm{hr}$ prior to removal of the fetuses. Newborn animals were kept with their mothers. Fasting newborns were placed in an incubator with ambient temperature maintained at $32^{\circ}-34^{\circ}$. Fasted newborns were returned to their mothers for refeeding.

Fetuses were delivered between 15 and 21 days of gestation by cesarean section. The fetuses in each litter were counted, weighed, and killed by decapitation. The liver was removed and weighed; four-six fetal livers were pooled. Newborn rats were sacrificed at specified intervals after birth. Their liver and spleen, and maternal liver and spleen, were removed and weighed individually. All tissues were homogenized in $2-5$ volumes $(w / v)$ of $0.25 \mathrm{~m}$ sucrose. Postmitochondrial supernatant fluids were prepared by centrifugation at $12,000 \times g$ in a refrigerated centrifuge. This relatively low fractionation speed was employed to avoid the loss of microsomes from immature liver [5]. Protein was determined by the method of Lowry et al. [9].

For the assay of microsomal heme oxygenase, a Gilford Model 2000 spectrophotometer, equipped with automatic recorder and constant temperature cuvette chamber, was used. The standard reaction mixture $(3.0$ ml) contained postmitochondrial supernatant fluid (3-15 mg protein); hemin, $17 \mu \mathrm{M}$; reduced nicotinamide adenine dinucleotide (NADPH), $180 \mu \mathrm{M}$; and potassium phosphate buffer ( $\mathrm{pH} 7.4$ ), $90 \mathrm{~mm}$. In the control cuvette, NADPH was replaced by $0.1 \mathrm{ml}$ of the phosphate buffer, pH 7.4. Formation of bilirubin was followed spectrophotometrically at $468 \mathrm{~m}_{\mu}$, the wavelength at which the pigment absorbs maximally in the incubation mixture [16]. The millimolar extinction coefficient of bilirubin at this wavelength ranges from 27.7 to 31.7 [16]. Enzyme activities were calculated from the linear portion of the spectral absorption curve. Microsomal yield was monitored with NADPHcytochrome $\mathrm{C}$ reductase activity in whole homogenates and postmitochondrial supernatant fluids [11].

\section{Results}

Hepatic heme oxygenase was highly active on the 15 th day of gestation, the earliest stage at which sufficient material could be obtained for examination. Fetal hepatic enzyme levels were relatively constant during later development (Fig. 1). A rapid increase occurred during the first postnatal day, followed by a more gradual rise to a maximum at 7 days of age. Activity then began to decline, reaching basal values at weaning. During the entire developmental period from the 15th day of gestation to weaning, hepatic heme oxygenase was more active in developing liver than in the liver of mature animals.

Hepatic and splenic heme oxygenase activities before and after birth are compared in Table I. Splenic activity followed a developmental pattern which was unlike that observed in the liver. Heme oxygenase in spleen was relatively stable during the first $24 \mathrm{hr}$ after birth, while activity was rising rapidly in liver. Activity in the spleen declined and activity in the liver continued to rise slowly during the 1st week of life. Thereafter, hepatic enzyme activity declined, and splenic enzyme activity increased three- to four-fold, reaching adult values at the time of weaning.

Fasting of pregnant rats between the 14th and 17th day of gestation, and between the 18th and 21st day of gestation, was associated with significantly $(P<0.05)$ increased hepatic heme oxygenase activities in fetuses and newborn offspring (Table II). The spleens of newborns and mothers were not affected by fasting. Fetal spleen provided too little tissue for analysis.

In newborn rats prevented from nursing, i.e., fasting, hepatic heme oxygenase activity increased $50 \%$ in $3 \mathrm{hr}$ and doubled within $6 \mathrm{hr}$ when compared with fed control newborns (Table III). Longer periods of starvation resulted in severe dehydration and death of newborn rats. When newborns starved for $3 \mathrm{hr}$ were allowed to suckle, hepatic heme oxygenase declined to control values by $6 \mathrm{hr}$ of age. The increase in activity during fasting and the decrease in activity which followed dietary reconstitution were statistically significant $(P<0.05)$. The activity of splenic enzyme remained unchanged during periods of fasting and refeeding. 


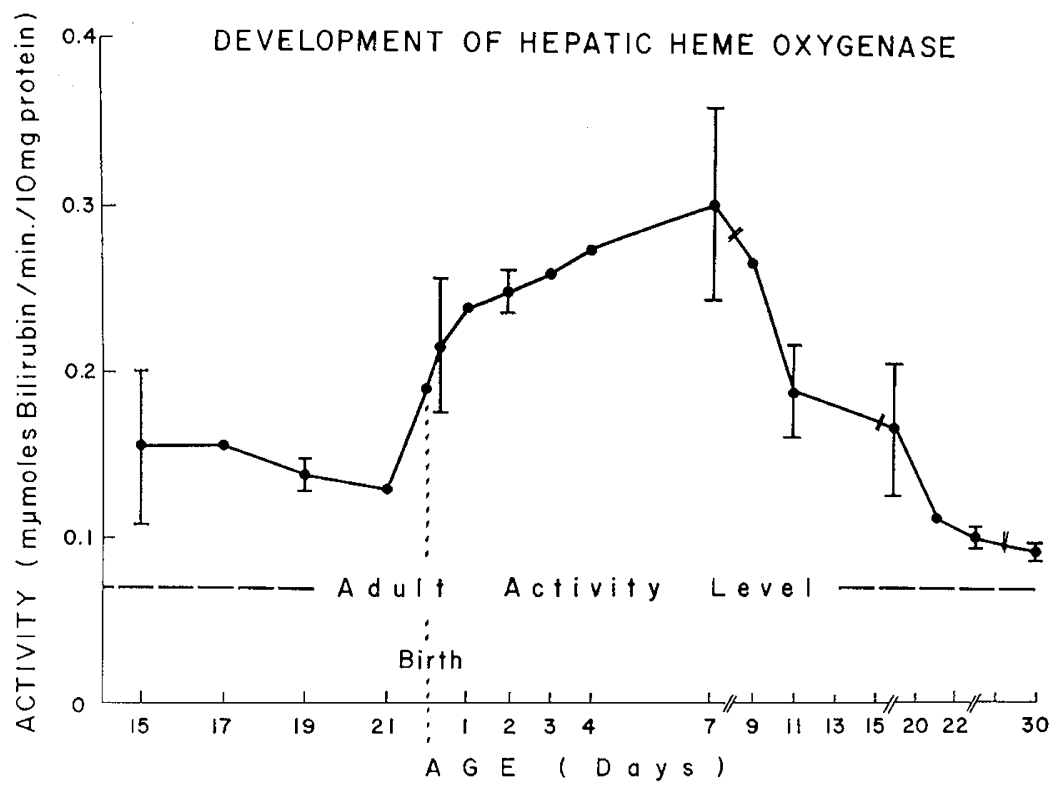

Fig. 1. Development of hepatic heme oxygenase activity from late gestation to weaning. Brackets represent standard deviations from the mean.

Table I. Heme oxygenase: perinatal activity

\begin{tabular}{|c|c|c|c|c|c|}
\hline & \multirow{2}{*}{ Age } & \multicolumn{2}{|c|}{ Liver } & \multicolumn{2}{|c|}{ Spleen } \\
\hline & & Absolute ${ }^{1}$ & Relative $^{2}$ & Absolute ${ }^{1}$ & Relative $^{2}$ \\
\hline Fetus & 21 days & $0.139 \pm 0.054$ & 199 & $0.430 \pm 0.080$ & 55 \\
\hline \multirow[t]{7}{*}{ Newborn } & $0 \mathrm{hr}$ & $0.190 \pm 0.057$ & 271 & $0.420 \pm 0.087$ & 54 \\
\hline & $6 \mathrm{hr}$ & $0.215 \pm 0.040$ & 307 & $0.440 \pm 0.096$ & 56 \\
\hline & $24 \mathrm{hr}$ & $0.237 \pm 0.040$ & 339 & $0.463 \pm 0.073$ & 59 \\
\hline & $48 \mathrm{hr}$ & $0.246 \pm 0.013$ & 350 & $0.383 \pm 0.065$ & 49 \\
\hline & $72 \mathrm{hr}$ & $0.257 \pm 0.054$ & 367 & $0.295 \pm 0.057$ & 37 \\
\hline & $96 \mathrm{hr}$ & $0.272 \pm 0.086$ & 389 & $0.206 \pm 0.063$ & 26 \\
\hline & Day 9 & $0.266 \pm 0.043$ & 380 & $0.272 \pm 0.036$ & 34 \\
\hline Weanling & Day 30 & $0.090 \pm 0.010$ & 129 & $0.860 \pm 0.160$ & 109 \\
\hline Adult & & $0.070 \pm 0.030$ & 100 & $0.790 \pm 0.130$ & 100 \\
\hline
\end{tabular}

${ }_{1}^{1}$ Expressed as millimicromoles bilirubin produced per minute per $10 \mathrm{mg}$ protein \pm standard deviation.

2 Expressed as percentage of adult activity.

Table II. Effect of 3-day maternal fasting on hepatic heme oxygenase in the newborn and fetal liver ${ }^{1}$

\begin{tabular}{lccr}
\hline & Fed & Fasted & $\begin{array}{c}\text { Increase, } \\
\%\end{array}$ \\
\hline Fetus (17 days) & $0.165 \pm 0.011$ & $0.202 \pm 0.018$ & 22 \\
Newborn & $0.170 \pm 0.013$ & $0.206 \pm 0.015$ & 18 \\
Mother & $0.060 \pm 0.010$ & $0.175 \pm 0.011$ & 190 \\
\hline
\end{tabular}

1 Expressed as millimicromoles bilirubin formed per minute per $10 \mathrm{mg}$ protein. A minimum of four litters was used for each determination.
Table III. Effects of fasting and refeeding on hepatic heme oxygenase in newborn rats

\begin{tabular}{rrrcc}
\hline \multirow{2}{*}{$\begin{array}{c}\text { Age, } \\
\mathrm{hr}\end{array}$} & $\begin{array}{c}\text { No. of } \\
\text { animals }\end{array}$ & \multicolumn{3}{c}{ Activity $^{1}$} \\
\cline { 3 - 5 } & \multicolumn{1}{c}{ Normal } & Fasted & Refed \\
\hline 0 & 12 & $0.23 \pm 0.03$ & & \\
3 & 6 & $0.29 \pm 0.08$ & $0.43 \pm 0.18$ & \\
6 & 5 & $0.32 \pm 0.12$ & $0.65 \pm 0.08$ & $0.28 \pm 0.07$
\end{tabular}

${ }^{1}$ Expressed as millimicromoles of bilirubin produced per minute per $10 \mathrm{mg}$ protein \pm standard deviation. 


\section{Discussion}

An understanding of the enzymic mechanisms involved in bilirubin production in fetuses and newborns should clarify the metabolic processes contributing to physiologic hyperbilirubinemia of the newborn. The metabolic system which converts heme to bilirubin consists of microsomal heme oxygenase, which catalyzes the oxidation of heme at the $\alpha$-methene bridge to form biliverdin, and biliverdin reductase, a soluble enzyme responsible for the reduction of biliverdin to bilirubin [16]. Inasmuch as both enzymes are present in the postmitochondrial supernatant fluid and both are NADPH-dependent, the total capacity of the pathway leading to bilirubin is tested with the standard heme oxygenase assay. However, heme oxygenase, its substrate heme, or biliverdin reductase may be limiting the stepwise conversion of heme to bilirubin.

Heme oxygenase activity seems to control the rate of bilirubin production in mature rat tissues, whereas biliverdin reductase is present in great excess in adult rat spleen and liver [18]. The reductase probably does not limit bilirubin production during fetal and perinatal development, inasmuch as the final product of enzymatic heme degradation is bilirubin rather than biliverdin at all ages examined. In addition, in vitro bilirubin production is greater in immature liver compared with mature liver, and was not enhanced in mixing experiments with purified microsomal suspensions as the source of heme oxygenase and with soluble proteins from adult rat liver and spleen as the source of biliverdin reductase [19]. These observations indicate that the reductase is at least as active in fetuses and newborns as in adults, and does not seem to limit bilirubin formation during late fetal and early postnatal development.

The supply of heme, rather than heme oxygenase activity, may regulate heme catabolism in the newborn or fetus. Recent experiments have shown, however, that this is not the case in adult rats [2]. Similar information concerning heme breakdown and bilirubin formation in rat fetuses and newborns is not available. In man, the removal of erythrocytes formed early in gestation provides increasing amounts of hemoglobin for catalysis during the last stages of fetal development. Betke [3] pointed out that the shortened red cell survival time and increased blood volume in the human fetus are associated with approximately 1.5fold rates of bilirubin production relative to body weight, compared with adults. Because heme stimulates hepatic, but not splenic, heme oxygenase activity [17], the high fetal hepatic heme oxygenase activity com- pared with relatively low splenic activity may reflect substrate induction of a rate-limiting enzyme. Additional stimulation may occur in the course of erythroblastosis fetalis by exposure to large amounts of heme in utero. In such cases, the fetal liver seems capable of producing an excess of bilirubin which, in the absence of conjugating activity [6], must be cleared by the placenta with extreme efficiency in order to prevent severe intrauterine hyperbilirubinemia.

Favorable conditions for active bilirubin formation also seem to be normally present at birth. In addition to a shortened red cell lifespan in newborns, Vest [21] has demonstrated that rates of bilirubin formation from nonerythrocyte sources were double and triple adult rates in normal newborns and premature infants, respectively. More recently, Maisels and his coworkers [10] showed that heme catabolism in newborn infants proceeded at twice adult rates. Consistent with increased rates of heme catabolism in newborn infants, heme oxygenase in the liver of rat fetuses and newborns is 2-3 times as active as in mature liver. Thus, whether enzyme or substrate is limiting, our results demonstrate that the enzymatic capacity for increased bilirubin production exists during fetal development and is enhanced further during the first 10 days after birth. In contrast to the high activity of heme oxygenase in newborn liver, glucuronyl transferase, the enzyme necessary for conjugation of bilirubin to an excretable product, is relatively inactive during the immediate postnatal period [4]. Physiologic hyperbilirubinemia of the newborn may, therefore, reflect increased production of bilirubin in an organism which is unable to excrete it.

The sudden rise in hepatic, but not splenic, heme oxygenase activity during the first $10 \mathrm{hr}$ after birth suggested that nutritional changes may play a role in the rapid postnatal activation of hepatic heme oxygenase. Greengard [7] has shown that many hepatic enzymes are activated at birth by hormones responsive to hypoglycemia or fasting. Recently, Bakken et al. [1] demonstrated that glucagon and epinephrine stimulate heme oxygenase in rat liver, whereas the spleen remains unresponsive. Because the newborn liver is depleted of glycogen, hypoglycemia develops rapidly when maternal supplies of glucose are interrupted. Fasting after birth resulted in rapid enhancement of hepatic heme oxygenase activity which was reversed when the newborns were allowed to suckle (Table III). A similar mechanism is probably responsible for the elevated activities found in fetuses and newborn offspring of rats fasted during pregnancy (Table II).

The rapid enlargement of the enzymatic capacity for 
conversion of heme to bilirubin, induced by postnatal starvation, may exceed the capacity of immature systems for bilirubin disposal, leading to retention of bilirubin in blood and tissues. Infants prone to hypoglycemia, such as prematures and infants of diabetic mothers, frequently develop severe hyperbilirubinemia during the 1st week of life $[8,14]$. Many reports suggest that, before early postnatal feeding became generally accepted in nurseries, jaundice was commonly observed in normal full term newborns whose first nourishment was withheld for $24 \mathrm{hr}$ or longer after birth $[13,22]$. Similarly, the unconjugated bilirubinemia in babies with pyloric stenosis [12] may reflect enhanced heme catabolism owing to the release of epinephrine and glucagon which follows rapid alterations in blood glucose concentrations [20].

\section{Summary}

Microsomal heme oxygenase, the system responsible for enzymatic conversion of heme to bilirubin, was assayed in liver and spleen of adult, newborn, and fetal rats under normal conditions and during periods of starvation. The results show that the liver possesses a higher capacity for enzymatic conversion of heme to bilirubin in fetal and newborn rats, whereas the spleen is the more active in adults. Maternal starvation, and fasting of offspring after birth, enhance the capacity for bilirubin production in the perinatal period. These observations indicate that a significant factor in jaundice of the newborn may be increased hepatic production of bilirubin, which may be further stimulated by starvation.

\section{References and Notes}

1. Bakken, A. F., Thaler, M. M., Prmstone, N., And Schmid, R.: Stimulation of hepatic heme oxygenase activity by fasting and by hormones. Gastroenterology, 60: 177 (1971).

2. Bakken, A. F., Thaler, M. M., and Schmid, R.: Hormonal control of hepatic heme catabolism. J. Clin. Invest., 50: 5a (1971).

3. Betke, K.: Bilirubin und Bilirubinausscheidung. Die Physiologische Entwicklung Des Kindes. (Springer-Verlag, Berlin, 1959).

4. BRown, A. K., ANd Zuelzer, W.: Studies on the neonatal development of the glucuronide conjugating system. J. Clin. Invest., 37: 332 (1958).

5. Chatterjee, I. B., Price, Z. H., and McKee, R. W.: Biosynthesis of L-ascorbic acid in different sub-cellular fractions of prenatal and postnatal rat livers. Nature, 207: 1168 (1965).

6. Dutron, G. J.: Comparison of glucuronide synthesis in developing mammalian and avian liver. Ann. N. Y. Acad. Sci., 111: 259 (1963).
7. GreEngard, O.: Enzymatic differentiation in mammalian liver. Science, 163: 891 (1969).

8. Hubbell, J. P., Drorbaugh, F. E., Rudolph, A. J., Auld, P. A. M., Cherry, R. B., AND SMith, C. A.: "Early" versus "late" feeding of infants of diabetic mothers. New Engl. J. Med., 265: 835 (1961).

9. Lowry, O. H., Rosebrough, N. J., FARR, A. L., AND RANDALL, R. J.: Protein measurement with the Folin phenol reagent. J. Biol. Chem., 193: 265 (1951).

10. Maisels, M. J., Pathak, A., Nelson, N. M., Nathan, D. G., AND SMrTh, C. A.: Endogenous production of carbon monoxide in normal and erythroblastotic newborn infants. $\mathrm{J}$. Clin. Invest., 50: I (1971).

11. Margoliash, E.: The chromatographic behaviour of cytochrome $c$ on cation exchangers. Biochem. J., 56: 535 (1954).

12. Marion, J., Daudet, M., and Freidel, M.: New data in intexpretation of jaundice accompanying hypertrophic pyloric stenosis. Pédiatrie, 22: 649 (1967).

13. Smallpeice, V., ANd Davies, P. A.: Immediate feeding of premature infants with undiluted breast-milk. Lancet, $i i$ : 1349 (1964).

14. Stimmler, L., Brazie, J. V., and O'Brien, D.: Plasma-insulin levels in newborn infants of normal and diabetic mothers. Lancet, $i$ : 137 (1964).

15. Tenhunen, R., Marver, H. S., and Schmid, R.: The enzymatic conversion of heme to bilirubin by microsomal heme oxygenase. Proc. Nat. Acad. Sci. U.S.A., 61: 748 (1968).

16. Tenhunen, R., Marver, H. S., and Schmid, R.: Microsomal heme oxygenase: characterization of the enzyme. J. Biol. Chem., 244: 6388 (1969).

17. Tenhunen, R., Marver, H. S., and Schmid, R.: Enzymatic catabolism of hemoglobin: stimulation of microsomal heme oxygenase by hemin. J. Lab. Clin. Med., 75: 410 (1970).

18. Tenhunen, R., Ross, M. E., Marver, H. S., and Schmid, R.: Reduced nicotinamide-adenine dinucleotide phosphate dependent biliverdin reductase: partial purification and characterization. Biochemistry, 9: 298 (1970).

19. Thaler, M. M., Gemes, D. L., And Bakken, A. F.: Unpublished results.

20. Unger, R. H., Ohneda, A., Aguilar-Parada, E., and EisenTRAUT, A. M.: The role of aminogenic glucagon secretion in blood glucose homeostasis. J. Clin. Invest., 48: 810 (1969).

21. Vest, M. F.: Studies on hemoglobin breakdown and incorporation of $\left[{ }^{15} \mathrm{~N}\right]$ glycine into heme and bile pigment in the newborn. In: I. A. D. Bouchier and B. H. Billing: Bilirubin Metabolism, p. 47. (F. A. Davis Company, Philadelphia, 1967).

22. Wu, P. Y. K., Teilmann, P., Gabler, M., Vaughan, M., and Metcoff, J.: "Early" versus "late" feeding of low birth weight neonates: effect on serum bilirubin, blood sugar and responses to glucagon and epinephrine tolerance tests. Pediatrics, 39: 733 (1967).

23. The authors wish to express their grateful appreciation to Dr. R. Schmid for crucial guidance and assistance.

24. Supported by Public Health Service Research Grant no. HD-03148.

25. Requests for reprints should be addressed to: M. Michael Thaler, M.D., Pediatric Department, University of California, San Francisco, California 94122.

26. Accepted for publication July $28,1971$. 